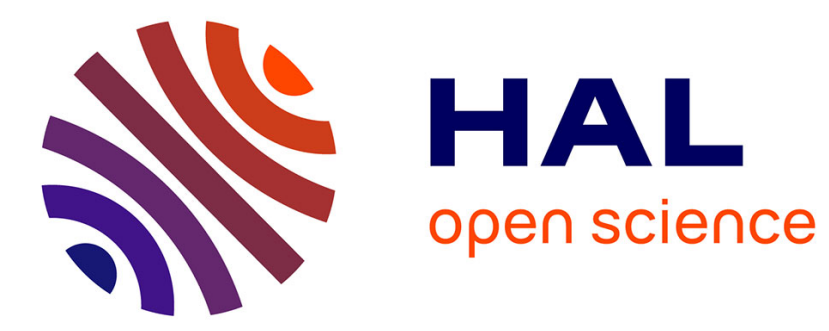

\title{
Fingerprint Quality Assessment With Multiple Segmentation
}

Z Yao, Jean-Marie Le Bars, Christophe Charrier, Christophe Rosenberger

\section{To cite this version:}

Z Yao, Jean-Marie Le Bars, Christophe Charrier, Christophe Rosenberger. Fingerprint Quality Assessment With Multiple Segmentation. International Conference on Cyberworlds, Oct 2015, Gotland - Sweeden, France. hal-01195799

\section{HAL Id: hal-01195799 \\ https://hal.science/hal-01195799}

Submitted on 8 Sep 2015

HAL is a multi-disciplinary open access archive for the deposit and dissemination of scientific research documents, whether they are published or not. The documents may come from teaching and research institutions in France or abroad, or from public or private research centers.
L'archive ouverte pluridisciplinaire HAL, est destinée au dépôt et à la diffusion de documents scientifiques de niveau recherche, publiés ou non, émanant des établissements d'enseignement et de recherche français ou étrangers, des laboratoires publics ou privés. 


\title{
Fingerprint Quality Assessment With Multiple Segmentation
}

\author{
Z. Yao, J-M. LeBars, C. Charrier and C. Rosenberger \\ Univeriste de Caen Basse Normandie; ENSICAEN. Caen, Calvados, France 14000
}

\begin{abstract}
Image quality is an important factor to automated fingerprint identification systems (AFIS) because the matching performance could be significantly affected by poor quality samples. Most of the existing studies mainly focused on calculating a quality index represented by either a single feature or a combination of multiple features, and some others achieve this purpose with learning approaches which may depend on a priorknowledge of matching performance. In this paper, a general framework for estimating fingerprint image quality is proposed by fusing features in segmentation phase. The quality index is indicated by a ratio of the pixel number of the integrated foreground area to the size (pixel number) of the fingerprint image. The potential advantage of this framework is that it could be improved by integrating other segmentation approaches or quality features rather than fusing them in a more complicated manner. The experiment is performed with several fingerprint datasets created via different sensors. Experimental results obtained from a dual evaluation approach demonstrate the validity of the proposed method in improving the overall performance.
\end{abstract}

\section{INTRODUCTION}

Fingerprint quality assessment (FQA) works as a toll-gate to ensure that poor quality samples could be rejected before sending them to next stage. This is very important to guarantee the performance of a biometric system [1], especially during the enrollment session. Therefore, this problem attracted attentions from both academic and industrial area, and a lot of studies had been made. Prior studies in estimating fingerprint quality could be classified into several categories:

1) Assessment approaches that rely on segmentation tasks, which could be either implemented by dividing the foreground area into several classes [2], [3], [4] or carried out via an approximation of the informative regions by using minutiae template only [5].

2) Quality indexes represented by a single feature [4], [6] which could be indicated by either the feature itself or an observed regularity of the employed feature [7].

3) Solutions carried out by using multi-feature fusion which could be achieved via a linear fusion or classification and both of them might involve in a prior-knowledge of matching performance [8], [9].

In addition, studies proposed in recent years have made attempt by learning [10] a multi-layer neural network. The quality feature in [10] is also indicated by a regularity of a histogram obtained from the best-matching unit assigned to fingerprint block. Likewise, the quality index is also involved in a classification that relies on a prior-knowledge of genuine matching scores.

According to the literature above, one can note that approaches based on categorizing fingerprint image areas or an image feature are easily affected by the change of image specification. On the other hand, assessing fingerprint quality with weighted or linear fusion is limited by employed coefficients, while the performance of quality metrics based on regression or classification are largely dependent on the involved regression approaches or classifiers, and the accuracy of the employed prior-knowledge. Therefore, it is still a challenge to achieve a common good quality metric for images captured by multi-sensor, even the resolutions of them are quite close to each other. Because of this, one can neither claim that metrics based on multi-feature fusion is able to make the assessment more robust nor deduce that it is not easy to apply a single feature to images collected with various sensors.

In this case, this study proposes a new quality assessment framework based upon trimming foreground pixels of bad quality image as much as possible. Therefore, instead of using solutions presented above, this study made effort in generating a quality metric with multiple coarse segmentation. This framework is almost a two-step (or more) work which firstly performs one coarse segmentation to the fingerprint image and followed by another segmentation-like operation for a further pixel-removing. Finally, each of the segmentation results is simply used as a feature, which makes fusing features in segmentation phase possible.

The following of the paper is organized as follows: Section II presents the proposed framework in detail. Section III gives the description of an auxiliary operation of the employed metric evaluation approach. In section IV, the experimental results figured out via the given evaluation approach are presented in detail. Section V concludes the paper.

\section{QUALITY ASSESSMENT FRAMEWORK}

As the specialty of the biometric application, fingerprint quality is not only image distortion determination. The purpose of the FQA is to guarantee the reliability of the feature extracted from the image and hence benefits the matching performance. In this case, segmentation is initially a choice to determine the useful and reliable area of the ridge-valley pattern, which somehow indicates fingerprint's availability in a quantitative manner [5]. Existing studies of fingerprint segmentation also involves in pure feature-based approaches and solutions with learning algorithm [11], [12], [13]. The feature-based approaches is affected by image specification and some learning-based approaches rely on large size training set and may not be appropriate to quality assessment applications. Note that segmentation is not equivalent to quality assessment in this case. In addition, prior studies in segmentation-based quality assessment mostly focus on determining foreground 
block number in terms of one (or more) specific feature(s) or assigning a goodness value to a block [14], [2]. In this case, the quality assessment framework proposed in this study considers to perform segmentation-based operations in multi-task. This is able to ignore the coefficients problem required by fusionbased methods and takes the advantage of the segmentation approaches based on a single feature. Furthermore, this is not relevant to any prior-knowledge such as matching scores. The selected segmentation criteria are common schemes and each of them acts as a module which is possibly to be replaced and improved.

\section{A. Feature given by Morphology Segmentation}

The first step of the proposed framework is to obtain a measure fingerprint foreground area as we have just mentioned before. To do this, a coarse segmentation is adopted in this study, which is achieved via morphological processing of images. Such a processing mainly consists of two tasks: dilation and erosion. Fingerprint image is composed by parallel run ridge-valley pattern with relatively stable frequency. With this property, it is able to connect the edges formed by the ridge-valley pattern (Cf. figure 1).

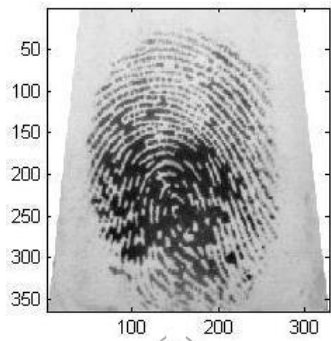

(a)

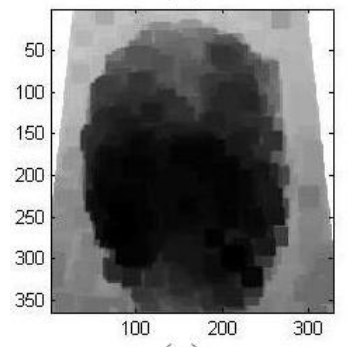

(c)

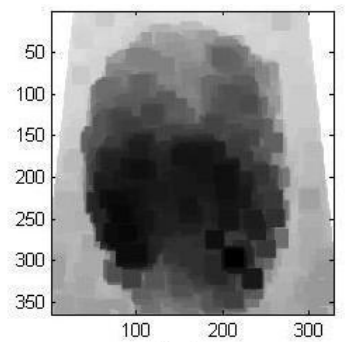

(b)

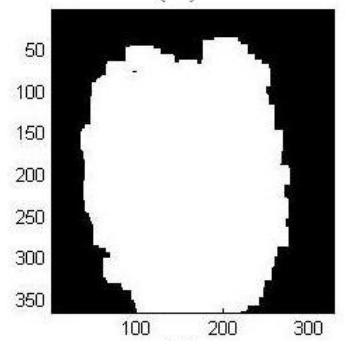

(d)
Fig. 1: Example of segmentation with morphology operation.

Four images in figure 1 illustrate a morphology processing of a fingerprint image with several iterations, where image 1(a) is the original fingerprint pattern, 1(b) is the image after erosion processing(s), 1(c) is the enhanced version of image $1(\mathrm{~b})$, and $1(\mathrm{~d})$ is the segmented mask. In this study, we use the approach in [15] to perform the first coarse segmentation. The first feature for indicating fingerprint quality is hence a pixel ratio of the foreground area to the entire image.

\section{B. Pixel-pruning based on Coherence}

In this task, we propose a pixel-pruning approach by using an existing segmentation criterion namely coherence [16]. The coherence is initially applied onto directional field estimation of fingerprint images and has been used as one of the features [16] for classification-based fingerprint segmentation approaches. The feature is to indicate the uniformity of the foreground gradients. In our experiments, we found that this feature is sensitive to the variation of the ridge-valley direction in a local area. Because of this, in this study, we customize an approach by using this feature to extensively remove foreground pixels in a local region where the directional information of the ridge-valley pattern changes abruptly.

The definition of the coherence is given by gradient measures of pixel intensity. In a local window $W$, it is defined by

$$
\operatorname{Coh}=\frac{\sqrt{\left(G_{x x}-G_{y y}\right)^{2}+4 G_{x y}^{2}}}{G_{x x}+G_{y y}}
$$

where $G_{x x}=\sum_{W} G_{x}^{2}, G_{y y}=\sum_{W} G_{y}^{2}, G_{x y}=\sum_{W} G_{x} G_{y}$ and $\left(G_{x}, G_{y}\right)$ is the local gradient.

Figure 2 illustrates an example of the pixel-pruning result of a fingerprint image.

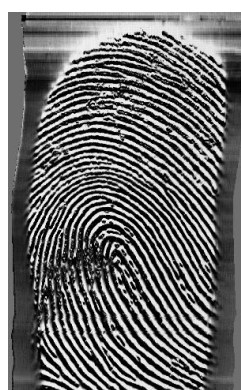

(a) Original

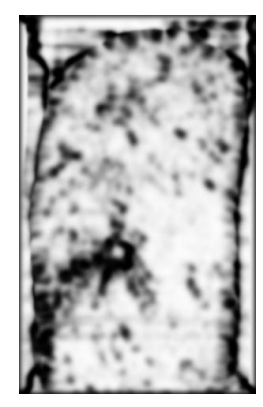

(b) Coherence

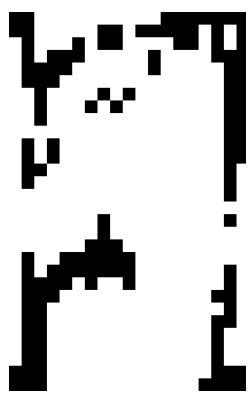

(c) Mask
Fig. 2: Example of segmentation with Coherence.

In figure 2, image 2(b) is the coherence image calculated from the original fingerprint illustrate by 2(a), while image 2(c) is the segmentation mask obtained by using our pixel-pruning method which is carried out via a thresholding operation to the coherence image.

In our study, the coherence image is first normalized into $[0,1]$, and then divided into non-overlapped blocks which is followed by thresholding operations with a baseline value of 0.5 . The block size is 16 in this study, and both the block size and threshold value are all empirical values in our study. Finally, the quality feature is also a ratio of the light pixels number to the pixel number of the entire image.

\section{Metric Generation}

The proposed framework of fingerprint quality assessment is essentially implemented by fusing two (or more) features in the segmentation phase, i.e. the binary images of mask obtained in the segmentation step and pixel-pruning session would be combined together. Considering score-based fusion in biometrics [17], one can observe that there are several ways to achieve fusion task such as 'min' and 'max' rules. In the proposed framework, we simply use the logical 'and' rule to fuse two binary mask images, which is actually equivalent to fusing two features (obtained by two steps) in terms of the 'add' rule. 
An example of such a fusion is given in figure 3 .

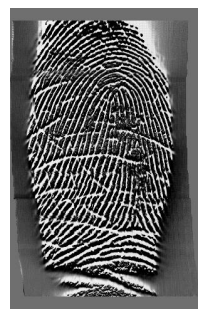

(a) Original

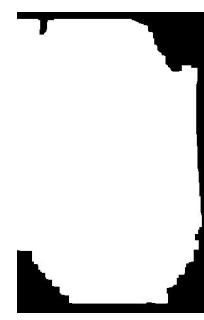

(b) Morphology

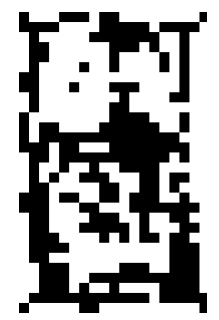

(c) Coherence (d) Fused

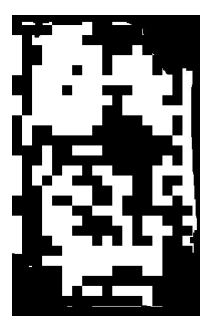

Fig. 3: Example of segmentation with Coherence

In figure 3 , one can note that the morphology approach is to coarsely generate an entire foreground area, where the pixelpruning approach is used for removing pixels in terms of the mean value of coherence in block-wise. The pruning task is particularly effective for bad quality images that contain some abrupt changes of the direction of the ridge-valley flow. The validation of the proposed approach is given by an evaluation approach, see section III.

\section{Evaluation}

The evaluation approach adopted in this study is based on the Enrollment Selection (ES) [18]. The ES measures a quality metric via a statistically computed global EER value, indicating the contribution of the quality metric in the degradation of the overall error rate.

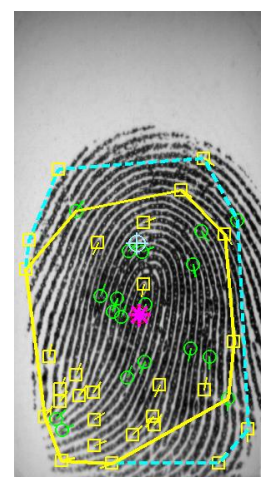

Fig. 4: Illustration of the disadvantage of using image center.

This section gives a description of an auxiliary means of the employed evaluation approach, which relies on the no-image minutiae selection (NIMS) [19]. The NIMS is an operation to reduce the size of minutiae template in terms of a desired number of minutiae points [19]. In this study, we use a selection criterion namely Vertex (abbr. as Vert) to remove minutiae. This criterion depends on the convex hull (polygon) of the minutiae template. The selection index is simply the distance between each vertex (Vert) minutia and the centroid of the convex hull of the minutiae template. First, the distance is simply calculated between each vertex and the centroid (pink star) of the polygon. The centroid of the polygon is used simply because the quality of an image is unknown and it is not appropriate to use the image center for some samples with only light translation of the foreground (even if the quality is not bad) (Cf. figure 4).

In figure 4, one can observe that some minutiae are relatively far from the image center (marked by cross ring) and removing these minutiae can lead to low genuine matching if the translation of another template is tiny or relatively smaller. For this measurement, with iteration operations, the vertices are pruned according to the largest value of the distances one by one. The desired number of selected minutiae ranges from 30 to 60 increased by 2 .

In this part, we firstly use the quality metrics calculating the quality value of each original sample, and then perform minutiae selection obtaining the reduced template and their matching scores. This operation generates 11 new datasets, where the template size of each dataset is fixed. After that, the ES operation is performed to each of the reduced dataset, detailed as:

1. The first sample of each individual is chosen as the enrollment to calculate a global EER for the original dataset. This global EER is denoted as 'NoSel'.

2. The ES operation is performed to each reduced dataset by using quality values of the original dataset. This operation hence computed another group of 11 global EER values for each reduced dataset. These values are able to measure the effect of quality to the NIMS. The effect is hence indicated by the difference between 'NoSel' and these 11 global EERs.

3. The ES is also performed to each reduced dataset by using the utility of the original database. This is to obtain a set of reference global EERs, for outlier is an unavoidable problem for the quality metrics.

Finally, a quality metric is validated via a comparison between 'NoSel' and global EERs obtained by step 2 .

\section{EXPERIMENTAL RESUlT}

The experiment given in this section includes two parts based on the Enrollment Selection (ES) [18]. At first, the simple ES associated to the proposed quality metric (denoted as MSEG hereinafter) and a reference metric (NFIQ) is performed with the original dataset. Second, the ES with the quality metrics is carried out via the auxiliary NIMS approach. The experiments employs several fingerprint databases and two different matching algorithms in this study. Details about employed software and datasets are given below. The MSEG is validated on five fingerprint datasets of the and two datasets re-organized from the CASIA database.

\section{A. Software}

In the experiment, we use two sets of software to calculate intra-class and inter-class matching scores for validating the MSEG. One set is the OpenSource NBIS software [20] and another is a commercial fingerprint SDK. The NBIS software consists of several modules, where the MINDTCT is an extractor generating INCITS 378-2004 standard minutiae template, and the Bozorth3 is used for calculating matching 
scores. The commercial SDK has 6 options of the existing minutiae template standards [21]. In the experiment, the minutiae templates of the ISO/IEC 19794-2:2005 standard have been extracted. A matcher implemented with the SDK is also employed to compute another group of matching scores, which enables validating the proposed MSEG within variant matching circumstances, i.e. an interoperate analysis is achieved.

\section{B. Database et Protocol}

In the experiment, we use several different datasets to perform the evaluation of the MSEG. First, five of Fingerprint Verification Competition (FVC) [22] database (Set A) are adopted, including FVC2000DB2, FVC2002DB2, and three of FVC2004 datasets. Second, we also generate two re-organized datasets from the CASIA FP-Test $\mathrm{V} 1^{1}$ database. Each of the FVC datasets includes 100 individuals and 8 samples per individual, 800 images in total. The CASIA database contains fingerprint images of 4 fingers of each hand of 500 subjects, where each finger has 5 samples. In this study, we create the two re-organized databases by using samples of the second finger of each hand, and they are respectively denoted as CASL2 and CASR2. Therefore, each sub-database has 2500 images of 500 individual ( 5 samples per individual).

The detail of each dataset is given in table I.

TABLE I: Dataset specification.

\begin{tabular}{|l|c|c|c|}
\hline DB & Sensor & Dim. & Resolution \\
\hline 00DB2A & Low-cost Capacitive & $256 \times 364$ & 500dpi \\
\hline 02DB2A & Optical & $296 \times 560$ & 569dpi \\
\hline 04DB1A & Optical & $640 \times 480$ & 500dpi \\
\hline 04DB2A & Optical & $328 \times 364$ & 500dpi \\
\hline 04DB3A & Thermal & $300 \times 480$ & 512dpi \\
\hline CASL & Optical & $328 \times 356$ & 512dpi \\
\hline CASR & Optical & $328 \times 356$ & 512dpi \\
\hline
\end{tabular}

The image size of each dataset is different from one another and the resolution is over 500dpi. A glance of the datasets are given by several samples in figure 5 .
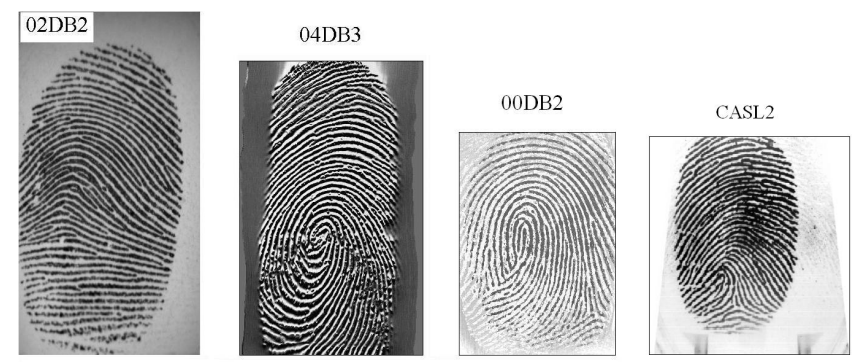

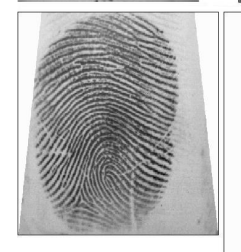

04DB2

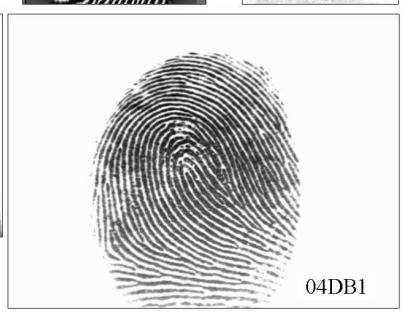

CASR2

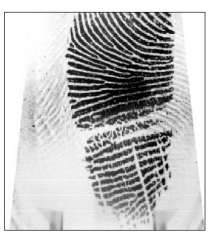

Fig. 5: Illustration of dataset samples.

\section{Results}

The experiment results are indicated by a set of global EER values and their $95 \%$ confidence interval (CI) obtained from each dataset by substituting the associated utility and quality values to the ES, respectively. The results obtained from FVC datasets and CASIA dataset are given separately, for the quality of these two classes are quite different.

Figure 6 plots the global EERs of the FVC datasets, where fig. 6 (a) is the result calculated from the NBIS matching scores and fig. 6 (b) shows the result obtained by using the matching scores of the SDK.

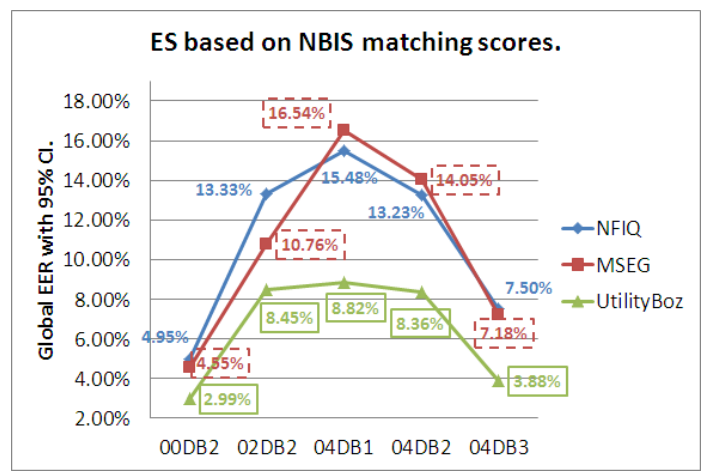

(a) Results based on NBIS software.

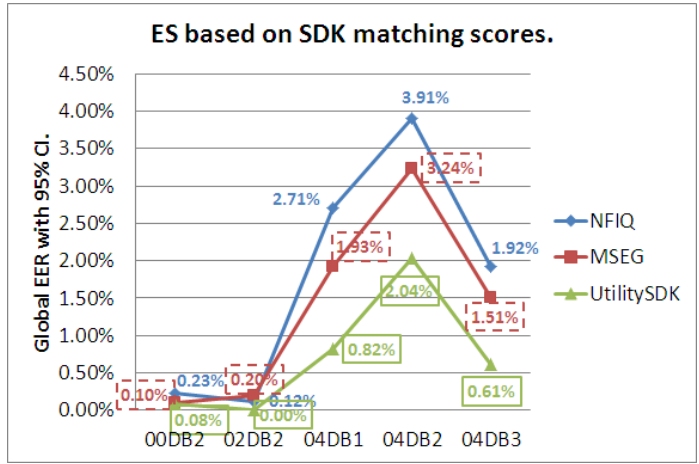

(b) Results based on SDK.

Fig. 6: Global EER plots. UtilityBoz and UtilitySDK in (a) and (b): global EER obtained with NBIS-based utility and SDK-based utility.

In fig. 6 (a), when NBIS matcher is involved, MSEG (red plot) respectively generates $16.54 \%$ and $14.05 \%$ on 04DB1 and 04DB2 which are relatively bad results in comparing with the reference metric (blue plot), while MSEG shows better results on the other 3 datasets. On the other hand, MSEG (fig. 6 (b)) performs relative bad on 02DB2 only and better on the other 4 datasets when a vendor-free matcher (SDK) is used. This is due to the difference of the matching performance between the two algorithms. In addition, the NFIQ is involved in a prior-knowledge of matching performance, which could more probably result in a different evaluation result. The global EERs of MSEG and NFIQ obtained from 02DB2 are $0.2 \%$ and $0.12 \%$, respectively. The global EERs obtained by samples utility are plotted via green points in each figure. The sample utility is simply an approximation of the groundtruth (with respect to the employed matcher) of the original sample [18].

\footnotetext{
${ }^{1}$ http://biometrics.idealtest.org/detailsDatabase.do?id=3
} 
TABLE II: The global EERs obtained from CASIA datasets.

\begin{tabular}{|c|c|c|c|c|}
\hline $\mathrm{QM} \quad \mathrm{DB}$ & CASL2 (NBIS) & CASR2 (NBIS) & CASL2 (SDK) & CASR2 (SDK) \\
\hline NFIQ & $43.09 \%$ & $43.51 \%$ & $40.92 \%$ & $38.20 \%$ \\
\hline MSEG & $42.30 \%$ & $43.20 \%$ & $38.61 \%$ & $35.97 \%$ \\
\hline
\end{tabular}

The utility-based global EERs are illustrated as a reference, indicating how much the quality metric is close to the best case that one matching algorithm can obtain from a trial dataset.

Table III gives the CIs of the quality-based global EERs, which has already included the CIs obtained from the two CASIA datasets.

The global EER values obtained by each quality metrics from the CASIA datasets are presented in table II.

According to the results given in table II, one can note that the proposed MSEG shows its validity in comparing with the reference quality metric. By using the matching scores of NBIS software, the global EERs obtained by MSEG are $42.30 \%$ (CASL2) and $43.20 \%$ (CASR2), while NFIQ generates $43.09 \%$ (CASL2) and $43.51 \%$ (CASR2), respectively. Likewise, the global EERs calculated with the matching scores of the SDK are $38.60 \%$ (CASL2), 35.97\% (CASR2), 40.92\%(CASL2) and $38.20 \%$ (CASR2), where the first two values correspond to the MSEG and the last two values belong to the NFIQ. The CIs given in table III are also consistent with these global EERs, indicating the validity of the proposed MSEG. Meanwhile, the experimental result also shows that the MSEG is commonly available for multiple image specifications, at least the employed image types.

TABLE III: The 95\% CI of the global EER of each metric.

\begin{tabular}{|c|c|c|}
\hline $\begin{array}{ll}\mathrm{DB} & \mathrm{QM} \\
\end{array}$ & NFIQ & MSEG \\
\hline 00DB2A (NBIS) & {$\left[\begin{array}{lll}0.0490 & 0.0500]\end{array}\right.$} & {$\left[\begin{array}{ll}0.0450 & 0.0461\end{array}\right]$} \\
\hline 02DB2A (NBIS) & {$\left[\begin{array}{lll}0.1326 & 0.1340\end{array}\right]$} & {$\left[\begin{array}{lll}0.1068 & 0.1084\end{array}\right]$} \\
\hline 04DB1A (NBIS) & {$\left[\begin{array}{lll}0.1540 & 0.1557\end{array}\right]$} & {$\left[\begin{array}{ll}0.1645 & 0.1662\end{array}\right]$} \\
\hline 04DB2A (NBIS) & {$\left[\begin{array}{lll}0.1312 & 0.1334\end{array}\right]$} & {$\left[\begin{array}{lll}0.1396 & 0.1413\end{array}\right]$} \\
\hline 04DB3A (NBIS) & {$\left[\begin{array}{ll}0.0745 & 0.0756\end{array}\right]$} & {$\left[\begin{array}{ll}0.0712 & 0.0723\end{array}\right]$} \\
\hline CASL2 (NBIS) & {$\left[\begin{array}{lll}0.4296 & 0.4322\end{array}\right]$} & {$\left[\begin{array}{ll}0.4213 & 0.4247\end{array}\right]$} \\
\hline CASR2 (NBIS) & {$\left[\begin{array}{ll}0.4337 & 0.4364\end{array}\right]$} & {$\left[\begin{array}{ll}0.4307 & 0.4332\end{array}\right]$} \\
\hline 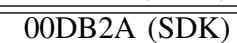 & 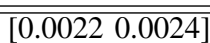 & 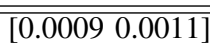 \\
\hline 02DB2A (SDK) & {$\left[\begin{array}{ll}0.0011 & 0.0013\end{array}\right]$} & {$\left[\begin{array}{ll}0.0019 & 0.0021\end{array}\right]$} \\
\hline 04DB1A (SDK) & {$\left[\begin{array}{lll}0.0266 & 0.0275\end{array}\right]$} & {$\left[\begin{array}{lll}0.0189 & 0.0196\end{array}\right]$} \\
\hline 04DB2A (SDK) & {$\left[\begin{array}{ll}0.0384 & 0.0397\end{array}\right]$} & {$\left[\begin{array}{ll}0.0319 & 0.0328\end{array}\right]$} \\
\hline 04DB3A (SDK) & {$\left[\begin{array}{ll}0.0189 & 0.0195\end{array}\right]$} & {$\left[\begin{array}{ll}0.0148 & 0.0153\end{array}\right]$} \\
\hline CASL2 (SDK) & {$\left[\begin{array}{ll}0.4087 & 0.4097\end{array}\right]$} & {$\left[\begin{array}{lll}0.3856 & 0.3866\end{array}\right]$} \\
\hline CASR2 (SDK) & {$\left[\begin{array}{ll}0.3815 & 0.3825\end{array}\right]$} & {$\left[\begin{array}{ll}0.3592 & 0.3603\end{array}\right]$} \\
\hline
\end{tabular}

Second, we perform validation of the MSEG by using the auxiliary approach mentioned in section III. This kind of operation needs to perform on one dataset for each desired number. Therefore, we simply choose one database as an example to illustrate this auxiliary method. In the experiment, the 04DB1 is used since there is a dissent between two matchers, and matching performance with the original template (NoSel) of this database is far from the global EERs obtained by utility-based ES, which makes a clear illustration. The plots of the global EERs of the reduced datasets are given in fig. 7.

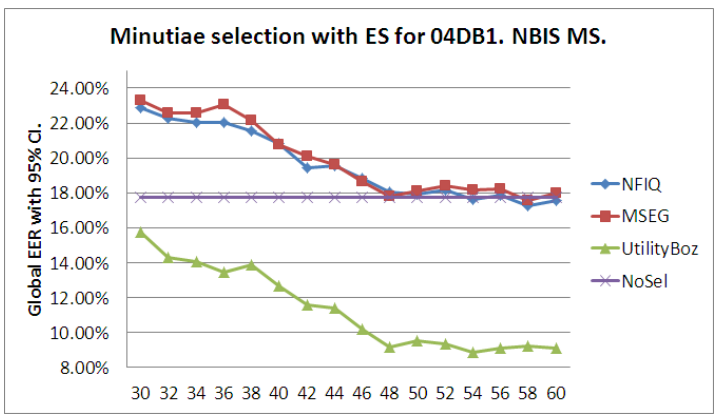

(a) Result based on NBIS matching scores.

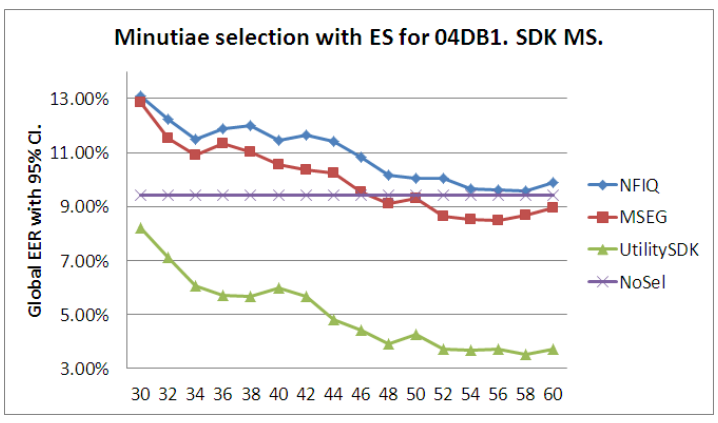

(b) Result based on SDK matching scores.

Fig. 7: Global EER plot obtained by ES of reduced templates.

In fig. 7 (a) and (b), 'UtilityBoz' and 'UtitlitySDK' respectively indicate global EERs' plots obtained via NBIS-based utility and SDK-based utility for each reduced template set. Obviously, by comparing the plots of the global EER values associated to the metrics, NFIQ (blue) and MSEG (red), the result is basically consistent with the ones given in fig. 6. A little bit variation appears as the desired number increased to 48 or so when calculating the global EER with NBIS matching scores. This is reasonable according to the study of NIMS [19]. In this case, with the reference quality metric and the objective measure (utility), one can found that the proposed framework is a valid solution for assessing fingerprint quality.

An implementation of the proposed framework is available online $^{2}$.

\section{CONCLUSion}

In this study, a new framework for qualifying fingerprint image is proposed. The proposed solution achieves fusing multi-feature in the segmentation phase, which avoids coefficient problem of the weight-based combination approaches. In addition, the proposed framework shows some generalities to variant fingerprint images. Finally, this approach is not related to any prior-knowledge, which makes the evaluation more independent.

\footnotetext{
${ }^{2}$ https://www.greyc.fr/users/zyao
} 
The validity of the proposed framework is proved via a comparison with the reference metric. The proposed quality assessment framework also has a potential advantage in improvement by using any available feature of fingerprint segmentation. Nevertheless, it could be integrated into the other quality assessment framework with multiple features as well.

\section{REFERENCES}

[1] P. Grother and E. Tabassi, "Performance of biometric quality measures," Pattern Analysis and Machine Intelligence, IEEE Transactions on, vol. 29, no. 4, pp. 531-543, 2007.

[2] R. M. Bolle, S. U. Pankanti, and Y. Yao, "System and method for determining the quality of fingerprint images," Oct. 5 1999, uS Patent $5,963,656$.

[3] L. Shen, A. Kot, and W. Koo, "Quality measures of fingerprint images," in IN: PROC. AVBPA, SPRINGER LNCS-2091, 2001, pp. 266-271.

[4] B. Lee, J. Moon, and H. Kim, "A novel measure of fingerprint image quality using the Fourier spectrum," in Society of Photo-Optical Instrumentation Engineers (SPIE) Conference Series, ser. Society of PhotoOptical Instrumentation Engineers (SPIE) Conference Series, A. K. Jain and N. K. Ratha, Eds., vol. 5779, Mar. 2005, pp. 105-112.

[5] Z. Yao, J.-M. LeBars, C. Charrier, and C. Rosenberger, "Quality assessment of fingerprints with minutiae delaunay triangulation," in International Conference on Information Systems Security and Privacy, Feb. 2015.

[6] Y. Chen, S. C. Dass, and A. K. Jain, "Fingerprint quality indices for predicting authentication performance," in Audio-and Video-Based Biometric Person Authentication. Springer, 2005, pp. 160-170.

[7] S. Lee, H. Choi, K. Choi, and J. Kim, "Fingerprint-quality index using gradient components," Information Forensics and Security, IEEE Transactions on, vol. 3, no. 4, pp. 792-800, 2008.

[8] M. El Abed, A. Ninassi, C. Charrier, and C. Rosenberger, "Fingerprint quality assessment using a no-reference image quality metric," in European Signal Processing Conference (EUSIPCO), 2013, p. 6.

[9] E. Tabassi, C. Wilson, and C. Watson, "NIST fingerprint image quality," NIST Res. Rep. NISTIR7151, 2004.

[10] M. Olsen, H. Xu, and C. Busch, "Gabor filters as candidate quality measure for NFIQ 2.0," in Biometrics (ICB), 2012 5th IAPR International Conference on, 2012, pp. 158-163.

[11] A. M. Bazen and S. H. Gerez, "Segmentation of fingerprint images," in Proc. Workshop on Circuits Systems and Signal Processing (ProRISC 2001). Citeseer, 2001, pp. 276-280.

[12] S. Chikkerur, C. Wu, and V. Govindaraju, "A systematic approach for feature extraction in fingerprint images," in Biometric Authentication. Springer, 2004, pp. 344-350.

[13] J. Yin, E. Zhu, X. Yang, G. Zhang, and C. Hu, "Two steps for fingerprint segmentation," Image and Vision Computing, vol. 25, no. 9, pp. 13911403, 2007.

[14] L. Hong, Y. Wan, and A. Jain, "Fingerprint image enhancement algorithm and performance evaluation," Pattern Analysis and Machine Intelligence, IEEE Transactions on, vol. 20, no. 8, pp. 777-789, 1998.

[15] "Fingerprint enhancement using STFT analysis," Pattern Recognition, vol. 40, no. 1, pp. $198-211,2007$.

[16] A. M. Bazen and S. H. Gerez, "Systematic methods for the computation of the directional fields and singular points of fingerprints," Pattern Analysis and Machine Intelligence, IEEE Transactions on, vol. 24, no. 7, pp. 905-919, 2002.

[17] A. Jain, K. Nandakumar, and A. Ross, "Score normalization in multimodal biometric systems," Pattern recognition, vol. 38, no. 12, pp. 2270-2285, 2005.

[18] Z. Yao, C. Charrier, and C. Rosenberger, "Utility validation of a new fingerprint quality metric," in International Biometric Performance Conference 2014. National Insititute of Standard and Technology (NIST), April 2014.
[19] B. Vibert, J.-M. Le Bars, C. Charrier, and C. Rosenberger, "Comparative study of minutiae selection algorithms for iso fingerprint templates," in Media Watermarking, Security, and Forensics XVI, ser. SPIE, February 2015.

[20] C. I. Watson, M. D. Garris, E. Tabassi, and etc., "User's guide to nist biometric image software (nbis)," 2007.

[21] O. for Standardization., "Iso/iec 19794-2:2005: Information technology. biometric data interchange formats-part 2: Finger minutiae data," 2005.

[22] D. Maio, D. Maltoni, R. Cappelli, J. L. Wayman, and A. K. J., "Fvc2004: Third fingerprint verification competition," in Biometric Authentication. Springer, 2004, pp. 1-7. 